\title{
The prevalence, awareness, treatment, and control of dyslipidemia in northeast China: a population-based cross-sectional survey
}

Fu-Liang Zhang ${ }^{1}$, Ying-Qi Xing ${ }^{2}$, Yan-Hua Wư ${ }^{3}$, Hao-Yuan Liu', Yun Luo', Ming-Shuo Sun ${ }^{1}$, Zhen-Ni Guo ${ }^{2 *}$ and Yi Yang ${ }^{1^{*}}$

\begin{abstract}
Background: Dyslipidemia is an important independent modifiable risk factor for cardiovascular disease. The aim of this study was to explore the current prevalence, awareness, treatment and control of dyslipidemia and its associated influence factors in northeast China.

Methods: In this population-based cross-sectional study, we adopted a multi-stage, stratified sampling method to obtain a representative sample of 4052 permanent residents aged 40 years and over from different urban and rural regions in Dehui City of Jilin Province. All subjects completed a questionnaire and were examined for risk factors. Continuous data were presented as means \pm standard deviations $(\mathrm{SD})$ and compared using the Student's $t$-test. Categorical variables were presented as proportions and compared using the Rao-Scott- $\chi^{2}$ test in different subgroups. The associated influence factors for the prevalence, awareness, treatment and control of dyslipidemia were evaluated through multivariate logistic regression.

Results: The prevalence of dyslipidemia was $62.1 \%$ overall, with $33.5,43.9,0.6$, and $8.8 \%$ for high total cholesterol, triglyceride, low-density lipoprotein cholesterol, and low high-density lipoprotein cholesterol, respectively. Among those with dyslipidemia, the proportion of subjects who were aware, treated, and controlled was 14.4, 33.9, and 19.9\%, respectively. Overweight or obesity ( $\mathrm{OR}=2.156 ; 95 \% \mathrm{Cl}: 1.863,2.533)$, hypertension $(\mathrm{OR}=1.643 ; 95 \% \mathrm{Cl}: 1.425,1.893)$, or diabetes mellitus $(\mathrm{OR}=2.173 ; 95 \% \mathrm{Cl}: 1.661,2.844)$ increased the prevalence of dyslipidemia, also these participants were more likely to be aware of their condition, however, this did not increase the likelihood of treatment and control. Living in urban areas and higher education level also increased the awareness of dyslipidemia. Personal history of coronary heart disease was the strongest influence factors associated with better awareness, treatment and control of dyslipidemia. Overweight or obesity $(\mathrm{OR}=0.404 ; 95 \% \mathrm{Cl}: 0.235,0.695)$ and lack of exercise $(\mathrm{OR}=0.423 ; 95 \% \mathrm{Cl}: 0.215,0$. 830) were associated with poor control of dyslipidemia.
\end{abstract}

Conclusion: The prevalence of dyslipidemia among adults aged 40 years and over in northeast China was high, however, the awareness, treatment, and control of dyslipidemia was measured at far from desirable levels. Renewed efforts taking influence factors into account are needed to improve the current unsatisfactory condition.

Keywords: Dyslipidemia, Prevalence, Awareness rate, Treatment rate, Control rate, Epidemiology, Northeast China

\footnotetext{
*Correspondence: zhen1ni2@163.com; doctoryangyi@163.com

${ }^{2}$ Neuroscience Center, Department of Neurology, the First Hospital of Jilin

University, Xinmin Street 71\#, 130021 Chang Chun, China

'Stroke Center, Department of Neurology, the First Hospital of Jilin University,

Xinmin Street 71\#, 130021 Changchun, China

Full list of author information is available at the end of the article
} 


\section{Background}

Coronary heart disease and cerebrovascular disease, as two major types of cardiovascular disease (CVD), were ranked as the top two caused of premature death and disability-adjusted life-years (DALYs) worldwide [1, 2], and contributed considerably to the increasing healthcare expenditures, especially in low- and middle-income countries, as well as in China [3, 4]. With the rapid economic expansion and increasing average life expectancy, CVD has become the leading cause of death in China, and its morbidity and mortality has been increasing since the late 1980s. Currently, CVD accounts for up to $40 \%$ of all deaths in both urban and rural populations in China [5].

On a more positive note, a considerable proportion of this morbidity and mortality could be prevented through early intervention of cardiovascular risk factors, such as low physical activity, tobacco use, dyslipidemia, hypertension, diabetes, etc.[6, 7], in which dyslipidemia plays an important role in the formation and development of atherosclerosis, and its association with risk of CVD is indisputable [8-10]. Therefore, early screening and effective lipid management may substantially reduce the burden of CVD and provide great social value [3].

With the improved standard of living, unhealthy dietary patterns and sedentary lifestyles have increased, and the age-standardized prevalence rate of dyslipidemia has been gradually increasing $[3,11,12]$. Moreover, this rate varies by region due to different climatic environments, diets, and lifestyles [13]. The latest data from Jilin Province in northeast China reported that the awareness, treatment, and control rate of dyslipidemia were 11.6, 8.4, and 34.8\%, respectively, in 2012 [14]. However, this data did not contain the prevalence rates of dyslipidemia or details regarding the subtypes of lipid disorders (high serum total cholesterol, triglycerides, low-density lipoprotein cholesterol, and low high-density lipoprotein cholesterol). The 2015-16 Stroke Screening and Prevention Program of the National Health and Family Planning Commission of China Study includes these data and remains the latest cross-sectional survey in northeast China. Based on this survey database, we analyzed the current prevalence, awareness, treatment, and control of dyslipidemia and its associated influence factors in Jilin Province, northeast China.

\section{Methods}

\section{Data source and study population}

This survey was conducted from January to March 2016 as part of the Stroke Screening and Prevention Program of the National Health and Family Planning Commission of China [15]. It was a population-based cross-sectional study designed to provide timely and reliable data for the prevalence of stroke and associated factors in adults over 40 years of age in northeast China, and it was supervised by the Chinese National Center for Stroke Care Quality Control and Management. This survey adopts the multistage stratified random cluster sampling method to select representative samples of the general population over 40 years who had lived in Dehui City for more than 6 months and voluntarily participated in the survey. In the first stage, 30 villages and 10 towns were selected from 308 villages (rural) and 14 towns (urban), respectively, in Dehui city. In the second stage, cluster sampling was used in the areas selected above. The study was approved by the Human Ethics and Research Ethics committees of the First Hospital of Jilin University (Approval Number: 2015-R-250). Written informed consent was obtained from all participants in the survey.

\section{Sampling size}

The targeted population for the Stroke Screening and Prevention Program of the National Health and Family Planning Commission of China 2015-16 were adults aged 40 years and over, and there were 335,490 residents over 40 years in Dehui City, according to the main data bulletin of the 6th National Population Census in 2010 (announced by the Statistical Bureau of Jilin Province). The expected sample size was 3355, which was $1 \%$ of the targeted population. Non-response rate was estimated as $20 \%$, so the planned sample size was 4026 ; a final number of 4100 residents participated in the survey. For the purpose of the present analysis, 48 subjects were excluded due to missing values. Finally, a total of 4052 people were included in the present analysis.

\section{Data collection and measurement}

All participants completed a structured pre-coded questionnaire designed by the National Health Development Planning Commission for Stroke Screening and Prevention Engineering, including demographic details (e.g., gender, age, residence, and education level), personal lifestyle (e.g., smoking, drinking, exercise habits, and diet structure), personal and family medical history of stroke and chronic diseases (i.e., hypertension, diabetes mellitus, dyslipidemia, and atrial fibrillation), and anthropometric measurements (e.g., height, weight, resting blood pressure, neck, waist, and hip circumference). Height and weight were measured according to standardized protocol and techniques, with the participants wearing clothes but no shoes. Blood pressure was measured by trained professionals using an electronic sphygmomanometer (OMRON HEM-7200), and each participant was measured twice, resting for at least $20 \mathrm{~min}$ before measurements were taken. We took the average of these readings. In addition, a blood sample was drawn from the participant's antecubital vein for measuring fasting blood glucose (FBG), total 
cholesterol (TC), triglycerides (TGs), low-density lipoprotein cholesterol (LDL-C), and high-density lipoprotein cholesterol (HDL-C), and the sample was uniformly measured by the Changchun Kingmed Center for Clinical Laboratory Co., Ltd. All participants underwent electrocardiograms for the detection of atrial fibrillation.

\section{Assessment criteria}

Dyslipidemia [16] was defined as using an antilipidemic medication or having one or more of the following in the field survey: TC $\geq 5.18 \mathrm{mmol} / \mathrm{L}, \mathrm{TGs} \geq 1.70 \mathrm{mmol} / \mathrm{L}$, $\mathrm{LDL}-\mathrm{C} \geq 3.37 \mathrm{mmol} / \mathrm{L}$, and. HDL-C $<1.04 \mathrm{mmol} / \mathrm{L}$.

Dyslipidemia awareness was defined as self-report of any previous diagnosis of dyslipidemia by a healthcare professional. The treatment rate of dyslipidemia was defined as the self-reported use of lipid-lowering drugs among participants who were aware of dyslipidemia. The control rate of dyslipidemia refers to the proportion among those treated for dyslipidemia who reach the lipid standard: $\mathrm{TG}<1.70 \mathrm{mmol} / \mathrm{L}, \mathrm{TC}<5.18 \mathrm{mmol} / \mathrm{L}$, $\mathrm{HDL}-\mathrm{C} \geq 1.04 \mathrm{mmol} / \mathrm{L}$, and LDL-C $<3.37 \mathrm{mmol} / \mathrm{L}$.

\section{Statistical analysis}

Complex weighted computation was used to make the sample more accurately representative of the population in Dehui City of the Jilin Province by post-stratification adjustment according to the following factors: age, residence, and gender groups, according to the standard population in the 6th national general investigation in Dehui City of the Jilin Province. Continuous data are presented as means \pm standard deviations $(\mathrm{SD})$ and compared using the Student's $t$-test. Categorical variables were presented as proportions and compared using the Rao-Scott- $\chi^{2}$ test in different subgroups. Finally, the associated influence factors for the prevalence, awareness, treatment, and control of dyslipidemia were analyzed through multivariate logistic regression. All statistical analyses were performed using the complex samples function of IBM SPSS 17.0 (SPSS Inc., New York, NY, USA). Statistical significance was set at $p<0.05$.

\section{Results}

\section{Characteristics of the study sample}

The study's 4052 participants included 1619 men (mean age was $55.72 \pm 9.43$ years) and 2433 women (mean age was $54.27 \pm 9.17$ years). The number of cases represented by urban participants was 2067 (51.0\%) and that of rural participants was 1985 (49.0\%).

Demographic and clinical characteristics of participants enrolled in this cross-sectional population-based survey according to dyslipidemia status are shown in Table 1. Compared with the non-dyslipidemia group, we found that the dyslipidemia group had a higher prevalence of hypertension, diabetes mellitus, overweight or obesity, lack of exercise, personal and family history of stroke and coronary heart disease, family history of dyslipidemia, and central obesity $(p<0.001$, except $p<0.05$ for family history of stroke). In addition to this, the dyslipidemia group was usually older and had higher systolic blood pressure and diastolic blood pressure, higher fasting blood-glucose, and higher neck, waist, and hip circumferences than those of the non-dyslipidemia group (all $p<0.001$ ).

\section{Prevalence of different types of dyslipidemia}

As shown in Table 2, the overall adjusted prevalence of dyslipidemia was $62.1 \%$ (95\% CI: 60.3-63.9\%). It first increased, and then decreased with age $(p<0.05)$, and the peak prevalence appeared in the 60-69 year age group, and the same trend occurred in high total cholesterol measurements $(p<0.001)$. The prevalence of dyslipidemia showed no statistically significant difference between males and females (61.3\% vs. $62.9 \%, p=0.383)$, but that of low high-density lipoprotein cholesterol was significantly higher in males than females $(11.1 \%$ vs. $6.4 \%, p<0.001$ ), yet the opposite was true for high total cholesterol (31.6\% vs. $35.5 \%, p=0.031)$. The adjusted prevalence rates of high total cholesterol, triglycerides, low-density lipoprotein, and low high-density lipoprotein were $33.5,43.9,0.6$, and $8.8 \%$, respectively. Compared with the rural areas, participants in urban areas had a higher prevalence of high triglyceride, low high-density lipoprotein, and dyslipidemia (all $p<0.001$ ). The prevalence of high total cholesterol decreased with education level $(p<0.001)$; however, that of low high-density lipoprotein showed the opposite trend $(p<0.001)$. With regard to body weight, the prevalence rates of dyslipidemia, high total cholesterol, triglycerides, low-density lipoprotein, and low high-density lipoprotein cholesterol were significant higher among those overweight or obesity than in others $(p<0.001$, except $p<0.05$ for high low-density lipoprotein). The prevalence of any types of dyslipidemia was not significantly associated with smoking or alcohol consumption status (all $p>0.05$ ). In addition, subjects who have personal or family history of stroke and coronary heart disease, or family history of dyslipidemia had higher prevalence of dyslipidemia than those without any history of these conditions (all $p<0.05$ ); and participants with family history of stroke were more likely to suffer from high lowdensity lipoprotein cholesterol compared to those without family history of stroke $(1.5 \%$ vs. $0.1 \%, p<0.001)$. Whereas, participants with family history of coronary heart disease or dyslipidemia had higher levels of prevalence of high total cholesterol and triglyceride as against those without any history of these conditions $(p<0.05)$.

\section{Awareness, treatment, and control of dyslipidemia}

The overall awareness, treatment, and control rates of dyslipidemia were 14.4\% (95\% CI: $12.9-16.0 \%$ ), 33.9\% 
Table 1 Characteristics of participants according to dyslipidemia status

\begin{tabular}{|c|c|c|c|c|}
\hline Characteristics & $\begin{array}{l}\text { Dyslipidemia } \\
(n=2570)\end{array}$ & $\begin{array}{l}\text { Normal } \\
(n=1482)\end{array}$ & $t$ & $p$ value \\
\hline Age (years) & $55.52 \pm 9.15$ & $53.68 \pm 9.45$ & 6.104 & $<0.001$ \\
\hline Gender (Male) & $1027(40.0)$ & $592(39.9)$ & & 0.992 \\
\hline Residence & & & & 0.037 \\
\hline Urban, n (\%) & $1343(52.3)$ & $724(48.9)$ & & \\
\hline Rural, n (\%) & $1227(47.7)$ & $758(51.1)$ & & \\
\hline Education, n (\%) & & & & 0.163 \\
\hline Primary school and below & $934(36.3)$ & $512(34.5)$ & & \\
\hline Junior middle school & 1070 (41.6) & $626(42.2)$ & & \\
\hline Senior middle school & $341(13.3)$ & $196(13.2)$ & & \\
\hline College and above & $225(8.8)$ & $148(10.0)$ & & \\
\hline a'Hypertension, n (\%) & $1668(64.9)$ & $667(45.0)$ & & $<0.001$ \\
\hline bDiabetes mellitus, n (\%) & $366(14.2)$ & $75(5.1)$ & & $<0.001$ \\
\hline 'Overweight or obesity, n (\%) & $962(37.4)$ & $277(18.7)$ & & $<0.001$ \\
\hline ¿Lack of exercise, n (\%) & $623(24.2)$ & 279 (18.8) & & $<0.001$ \\
\hline${ }^{\mathrm{e}}$ Personal history of stroke, $\mathrm{n}(\%)$ & $219(8.5)$ & $73(4.9)$ & & $<0.001$ \\
\hline fFamily history of stroke, n (\%) & $921(35.8)$ & $476(32.1)$ & & 0.016 \\
\hline${ }^{9}$ Personal history of coronary heart disease, n (\%) & $207(8.1)$ & $65(4.4)$ & & $<0.001$ \\
\hline${ }^{h}$ Family history of coronary heart disease, $n(\%)$ & $694(27.0)$ & $317(21.4)$ & & $<0.001$ \\
\hline 'Family history of dyslipidemia, n (\%) & $498(19.4)$ & $126(8.5)$ & & $<0.001$ \\
\hline BMI $\left(\mathrm{kg} / \mathrm{m}^{2}\right), \mathrm{n}(\%)$ & & & & $<0.001$ \\
\hline $\mathrm{BMI}<18.0$ & $25(1.0)$ & $39(2.6)$ & & \\
\hline $18 \leq \mathrm{BMl}<24.0$ & $944(36.7)$ & $884(59.6)$ & & \\
\hline $24 \leq \mathrm{BMl}<26.0$ & $639(24.9)$ & $282(19.0)$ & & \\
\hline $26 \leq \mathrm{BMl}<28.0$ & $497(19.3)$ & $154(10.4)$ & & \\
\hline $\mathrm{BMI} \geq 28.0$ & $465(18.1)$ & $123(8.3)$ & & \\
\hline 'Central obesity, n (\%) & $1516(59.0)$ & $528(35.6)$ & & $<0.001$ \\
\hline SBP $(\mathrm{mmHg})$ & $144.16 \pm 21.61$ & $135.58 \pm 20.73$ & 12.485 & $<0.001$ \\
\hline $\mathrm{DBP}(\mathrm{mmHg})$ & $90.46 \pm 11.91$ & $85.86 \pm 11.03$ & 12.416 & $<0.001$ \\
\hline \multicolumn{5}{|l|}{ TG CHOHDLLDL (mmol/L) } \\
\hline TC & $5.77 \pm 1.21$ & $4.75 \pm 0.68$ & 34.294 & $<0.001$ \\
\hline TG & $2.61 \pm 2.01$ & $1.12 \pm 0.31$ & 36.703 & $<0.001$ \\
\hline LDL-C & $2.16 \pm 0.77$ & $2.09 \pm 0.85$ & 2.2652 & 0.008 \\
\hline $\mathrm{HDL}-\mathrm{C}$ & $1.22 \pm 0.26$ & $1.31 \pm 0.20$ & -11.132 & $<0.001$ \\
\hline FBG (mmol/L) & $5.52 \pm 1.88$ & $4.98 \pm 1.08$ & 11.640 & $<0.001$ \\
\hline Neck circumference (cm) & $34.71 \pm 3.37$ & $33.40 \pm 2.94$ & 12.936 & $<0.001$ \\
\hline Waist circumference $(\mathrm{cm})$ & $87.51 \pm 8.86$ & $82.27 \pm 8.99$ & 18.035 & $<0.001$ \\
\hline Hip circumference (cm) & $97.77 \pm 8.25$ & $93.50 \pm 8.36$ & 15.737 & $<0.001$ \\
\hline Fruit consumption, n (\%) & & & & 0.998 \\
\hline$\geq 5 \mathrm{~d} / \mathrm{w}$ & $2273(88.4)$ & $1307(88.2)$ & & \\
\hline $3-4 d / w$ & $223(8.7)$ & $136(9.2)$ & & \\
\hline$\leq 2 \mathrm{~d} / \mathrm{w}$ & $74(2.9)$ & $39(2.6)$ & & \\
\hline
\end{tabular}


Table 1 Characteristics of participants according to dyslipidemia status (Continued)

\begin{tabular}{|c|c|c|c|}
\hline kSmoker & & & 0.231 \\
\hline Yes & $1304(50.7)$ & $723(48.8)$ & \\
\hline No & $1266(49.3)$ & 759 (51.2) & \\
\hline Alcohol consumption, n (\%) & & & 0.887 \\
\hline Never & $1878(73.1)$ & $1100(74.2)$ & \\
\hline Moderate drinking & $399(15.5)$ & $242(16.3)$ & \\
\hline Excessive drinking & $293(11.4)$ & $140(9.4)$ & \\
\hline \multicolumn{4}{|c|}{ 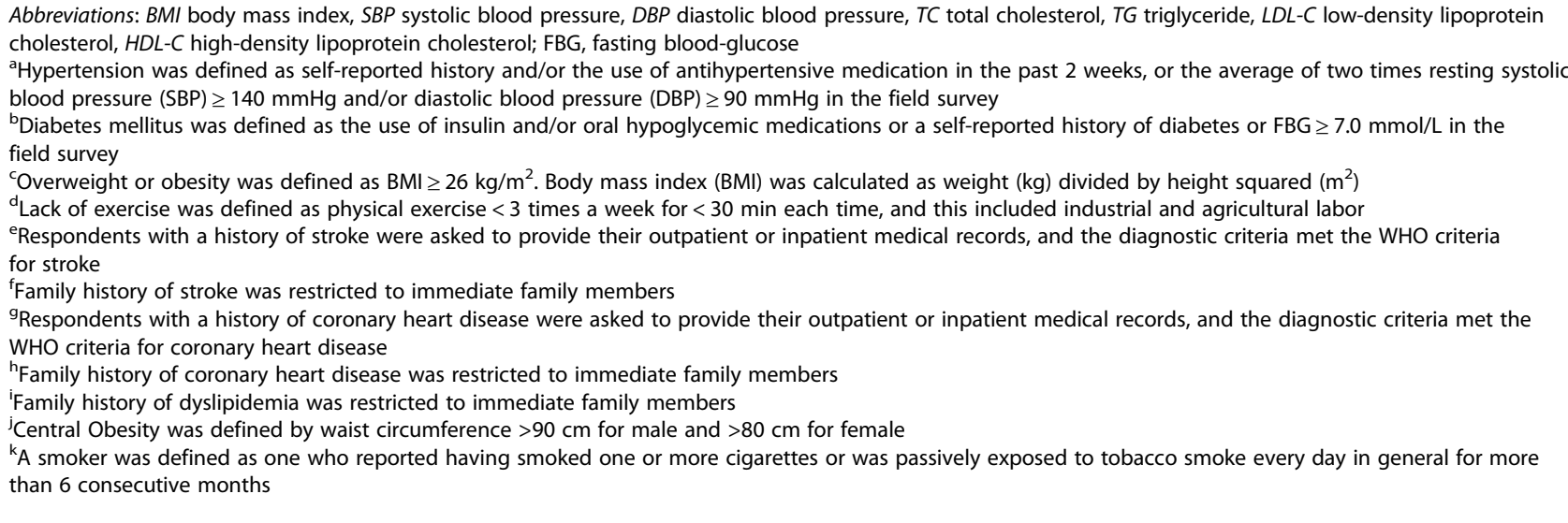 } \\
\hline
\end{tabular}

(95\% CI: $28.5-39.8 \%)$, and 19.9\% (95\% CI: 15.5-25.2\%), respectively (shown in Table 3 ).

\section{Awareness of dyslipidemia}

The awareness of dyslipidemia generally increased with increasing age $(p=0.007)$, with the last age group 70having the highest level of awareness, $18.9 \%$ (95\% CI: 13.3-26.0\%); additionally, those living in the urban areas, with a high educational level, and those who were lack of exercise, overweight or obesity, with personal history of stroke or coronary heart disease, and family history of coronary heart disease usually had a higher rate of awareness than those living in the rural areas, with a low educational level, and those who got enough exercise, had a relatively normal body mass index, without personal history of stroke or coronary heart disease, or family history of coronary heart disease (all $p<0.05$ ). However, the awareness of dyslipidemia was not significantly associated with gender $(p=0.287)$, smoking $(p=0.236)$ or alcohol consumption status $(p=0.185)$.

\section{Treatment of dyslipidemia}

Among participants who were aware of dyslipidemia, those with family history of coronary heart disease were more likely to treat their dyslipidemia than those without family history of coronary heart disease $(p=0.030)$. In the present study, treatment of dyslipidemia was not found to be significantly associated with age $(p=0.763)$, gender $(p=0.320)$, residential area $(p=0.237)$, education level $(p=0.225)$, exercise habits $(p=0.128)$, smoking $(p=0.245)$ or alcohol consumption status $(p=0.282)$, BMI $(p=0.476)$, personal history of stroke $(p=0.147)$ or coronary heart disease $(p=0.080)$, or family history of stroke $(p=0.122)$ or dyslipidemia $(p=0.224)$.

\section{Control of dyslipidemia}

Among dyslipidemia patients using lipid-lowering drugs, participants with regular exercise were more likely to have their dyslipidemia controlled than those who were lack of exercise (23.4\% vs. $10.2 \%, p=0.013)$. Besides, overweight or obesity (BMI $\geq 26 \mathrm{~kg} / \mathrm{m}^{2}$ ) was associated with a lower level of dyslipidemia control than those with $\mathrm{BMI}<26 \mathrm{~kg} / \mathrm{m}^{2}(p=0.006)$.

\section{Factors associated with the prevalence, awareness, treatment, and control of dyslipidemia}

Multiple logistic regression analysis (Table 4) suggests that the prevalence of dyslipidemia was significantly associated with increasing age; and participants living in urban regions $(\mathrm{OR}=1.339 ; 95 \% \mathrm{CI}: 1.161,1.543)$ were more inclined to suffer from dyslipidemia. Overweight or obesity (OR $=2.156 ; 95 \% \mathrm{CI}: 1.863,2.533)$ and lack of exercise (OR $=1.212$; 95\% CI: $1.021,1.439)$ were associated with an increased prevalence of dyslipidemia. Subjects with hypertension $(\mathrm{OR}=1.643 ; 95 \% \mathrm{CI}$ : 1.425 , 1.893), or diabetes mellitus $(\mathrm{OR}=2.173 ; 95 \% \mathrm{CI}: 1.661$, 2.844) were more likely to have dyslipidemia. In addition, participants with personal $(\mathrm{OR}=1.365$; 95\% CI: $1.006,1.851$ ) or family history of coronary heart disease $(\mathrm{OR}=1.182$; $95 \% \mathrm{CI}: 1.004,1.391)$, or family history of 
Table 2 Prevalence of different types of dyslipidemia in northeast China

\begin{tabular}{|c|c|c|c|c|c|c|}
\hline Category & Subcategory & $\mathrm{H}-\mathrm{TC}$ & $\mathrm{H}-\mathrm{TG}$ & $\mathrm{H}-\mathrm{LDL}$ & L-HDL & Dyslipidemia \\
\hline Total & & $33.5(31.8-35.3)$ & $43.9(42.0-45.7)$ & $0.6(0.3-1.0)$ & $8.8(7.8-9.8)$ & $62.1(60.3-63.9)$ \\
\hline \multirow[t]{5}{*}{ Age } & $40-49$ & $27.3(24.4-30.4)$ & $44.2(40.9-47.5)$ & $0.6(0.2-1.6)$ & $9.2(7.5-11.2)$ & $58.6(55.3-61.8)$ \\
\hline & $50-59$ & $37.3(34.5-40.2)$ & $45.5(42.6-48.5)$ & $0.5(0.2-1.4)$ & $8.6(7.1-10.3)$ & $64.4(61.4-67.2)$ \\
\hline & $60-69$ & $38.5(35.3-41.8)$ & $44.3(41.0-47.6)$ & $0.3(0.1-1.1)$ & 7.9(6.4-9.7) & $65.7(62.4-68.9)$ \\
\hline & $70-$ & $38.2(32.1-44.7)$ & $37.2(31.3-43.6)$ & $0.8(0.2-3.4)$ & $9.1(6.3-13.0)$ & $63.3(56.8-69.3)$ \\
\hline & $p$ value & $<0.001$ & 0.092 & 0.863 & 0.792 & 0.014 \\
\hline \multirow[t]{3}{*}{ Gender } & Male & $31.6(28.9-34.3)$ & $42.2(39.4-45.1)$ & $0.7(0.4-1.5)$ & $11.1(9.5-12.9)$ & $61.3(58.5-64.1)$ \\
\hline & Female & $35.5(33.2-37.8)$ & $45.6(43.2-47.9)$ & $0.4(0.1-0.9)$ & $6.4(5.4-7.6)$ & $62.9(60.6-65.2)$ \\
\hline & $p$ value & 0.031 & 0.075 & 0.236 & $<0.001$ & 0.383 \\
\hline \multirow[t]{3}{*}{ Residence } & Urban & $28.8(26.8-30.9)$ & 49.6(47.3-51.8) & $0.2(0.1-0.7)$ & $15.6(14.0-17.4)$ & $66.4(64.2-68.4)$ \\
\hline & Rural & $35.1(32.9-37.4)$ & $42.0(39.6-44.3)$ & $0.7(0.4-1.2)$ & $6.5(5.4-7.8)$ & $60.7(58.3-63.0)$ \\
\hline & $p$ value & $<0.001$ & $<0.001$ & 0.089 & $<0.001$ & $<0.001$ \\
\hline \multirow[t]{5}{*}{ Education } & Primary school and below & $36.8(34.1-39.6)$ & 43.7(40.9-46.6) & $0.6(0.3-1.2)$ & $6.9(5.6-8.6)$ & $62.9(60.0-65.6)$ \\
\hline & Junior middle school & $31.3(28.6-34.2)$ & $43.4(40.5-46.4)$ & $0.6(0.2-1.6)$ & $9.6(8.1-11.3)$ & $60.5(57.5-63.4)$ \\
\hline & Senior middle school & $30.7(26.3-35.5)$ & $44.7(39.9-49.7)$ & $0.2(0.0-1.7)$ & $12.6(9.6-16.3)$ & $64.2(59.3-68.9)$ \\
\hline & College and above & 23.6(19.1-28.9) & $47.7(42.1-53.4)$ & $0.4(0.1-3.1)$ & 13.6(10.3-17.8) & $63.9(58.5-69.0)$ \\
\hline & $p$ value & $<0.001$ & 0.685 & 0.878 & $<0.001$ & 0.391 \\
\hline \multirow[t]{3}{*}{ Lack of exercise } & Yes & $33.9(30.1-38.0)$ & $49.9(45.8-54.0)$ & $0.1(0.0-0.4)$ & $12.3(10.1-14.8)$ & $67.4(63.3-71.2)$ \\
\hline & No & $33.4(31.5-35.4)$ & $42.6(40.5-44.6)$ & $0.7(0.4-1.2)$ & $8.0(7.0-9.2)$ & 61.0(58.9-63.0) \\
\hline & $p$ value & 0.839 & 0.002 & 0.003 & $<0.001$ & 0.006 \\
\hline \multirow[t]{3}{*}{ Smoking } & Yes & $34.1(31.7-36.5)$ & $44.4(41.9-46.9)$ & $0.5(0.3-1.1)$ & $8.4(7.1-9.8)$ & $62.4(59.9-64.8)$ \\
\hline & No & $32.7(30.1-35.3)$ & 43.1(40.4-45.8) & $0.6(0.3-1.5)$ & $9.4(8.0-11.1)$ & $61.7(58.9-64.3)$ \\
\hline & $p$ value & 0.433 & 0.478 & 0.787 & 0.314 & 0.691 \\
\hline \multirow[t]{3}{*}{ Overweight or obesity } & Yes & $38.5(35.3-41.8)$ & $62.0(58.7-65.2)$ & $1.1(0.5-2.4)$ & 13.6(11.4-16.0) & 77.2(74.3-79.9) \\
\hline & No & $31.3(29.2-33.4)$ & $35.7(33.6-37.9)$ & $0.3(0.1-0.7)$ & $6.6(5.7-7.7)$ & $55.3(53.0-63.7)$ \\
\hline & $p$ value & $<0.001$ & $<0.001$ & 0.012 & $<0.001$ & $<0.001$ \\
\hline \multirow[t]{4}{*}{ Alcohol consumption } & Never & $33.7(31.7-35.8)$ & $43.9(41.8-46.0)$ & $0.5(0.2-1.0)$ & 8.7(7.6-9.9) & $62.0(59.9-64.1)$ \\
\hline & Moderate drinking & $31.4(27.2-35.9)$ & 43.5(39.0-48.2) & $0.7(0.2-2.4)$ & $9.4(7.1-12.4)$ & $61.2(56.6-65.7)$ \\
\hline & Excessive drinking & $35.7(30.6-41.2)$ & $44.4(38.9-50.0)$ & $0.6(0.2-2.6)$ & $8.4(6.0-11.6)$ & $64.1(58.5-69.4)$ \\
\hline & $p$ value & 0.438 & 0.973 & 0.859 & 0.814 & 0.712 \\
\hline \multirow[t]{3}{*}{ Personal history of stroke } & Yes & $38.5(32.2-45.3)$ & $50.9(44.1-57.6)$ & $0.1(0.0-0.8)$ & $11.5(7.9-16.5)$ & $73.5(67.1-79.0)$ \\
\hline & No & $33.1(31.3-35.0)$ & $43.3(41.4-45.3)$ & $0.6(0.3-1.1)$ & 8.6(7.6-9.6) & $61.2(59.3-63.1)$ \\
\hline & $p$ value & 0.109 & 0.035 & 0.078 & 0.137 & $<0.001$ \\
\hline \multirow[t]{3}{*}{ Family history of stroke } & Yes & $35.1(32.1-38.2)$ & $46.1(42.9-49.3)$ & $1.5(0.8-2.7)$ & 10.0(8.2-12.1) & $66.0(62.9-68.9)$ \\
\hline & No & $32.7(30.6-34.9)$ & $42.8(40.5-45.0)$ & $0.1(0.0-0.4)$ & $8.2(7.1-9.4)$ & $60.2(57.9-62.4)$ \\
\hline & $p$ value & 0.221 & 0.098 & $<0.001$ & 0.099 & 0.003 \\
\hline \multirow{3}{*}{$\begin{array}{l}\text { Personal history of } \\
\text { coronary heart disease }\end{array}$} & Yes & $27.8(22.3-34.1)$ & $50.7(43.6-57.7)$ & $0.3(0.0-2.4)$ & $12.1(8.4-17.2)$ & $69.6(62.4-76.0)$ \\
\hline & No & $33.9(32.1-35.8)$ & $43.4(41.5-45.3)$ & $0.6(0.3-1.0)$ & $8.5(7.6-9.6)$ & $61.6(59.7-63.5)$ \\
\hline & $p$ value & 0.066 & 0.051 & 0.618 & 0.075 & 0.035 \\
\hline \multirow{3}{*}{$\begin{array}{l}\text { Family history of coronary } \\
\text { heart disease }\end{array}$} & Yes & $38.2(34.6-41.9)$ & $47.2(43.4-50.9)$ & $0.8(0.3-2.3)$ & $8.8(6.9-11.2)$ & $67.6(64.0-71.1)$ \\
\hline & No & $32.0(30.1-34.1)$ & $42.8(40.7-45.0)$ & $0.5(0.2-0.9)$ & $8.8(7.7-10.0)$ & $60.4(58.2-62.5)$ \\
\hline & $p$ value & 0.003 & 0.049 & 0.427 & 0.938 & 0.001 \\
\hline \multirow[t]{3}{*}{ Family history of dyslipidemia } & Yes & $47.3(42.8-51.8)$ & 57.5(53.0-61.9) & $1.2(0.5-3.1)$ & $10.0(7.6-13.1)$ & 79.8(75.9-83.1) \\
\hline & No & $30.3(28.4-32.2)$ & $40.7(38.7-42.7)$ & $0.4(0.2-0.8)$ & $8.5(7.5-9.6)$ & $57.9(55.9-60.0)$ \\
\hline & $p$ value & $<0.001$ & $<0.001$ & 0.054 & 0.290 & $<0.001$ \\
\hline
\end{tabular}


Table 3 Awareness, treatment and control of dyslipidemia

\begin{tabular}{|c|c|c|c|c|}
\hline \multirow[t]{2}{*}{ Category } & \multirow[t]{2}{*}{ Subcategory } & \multicolumn{3}{|l|}{ Dyslipidemia } \\
\hline & & Awareness & Treatment & Control \\
\hline Total & & $14.4(12.9-16.0)$ & $33.9(28.5-39.8)$ & $19.9(15.5-25.2)$ \\
\hline \multirow[t]{5}{*}{ Age } & $40-49$ & $11.6(9.2-14.6)$ & $35.3(24.4-48.0)$ & $17.7(10.0-29.2)$ \\
\hline & $50-59$ & 13.7(11.5-16.3) & $29.8(22.2-38.7)$ & $14.6(9.0-22.7)$ \\
\hline & $60-69$ & 18.5(15.6-21.8) & $34.5(26.3-43.8)$ & $21.8(15.0-30.6)$ \\
\hline & $70-$ & $18.9(13.3-26.0)$ & $38.7(22.7-57.6)$ & $33.1(18.3-52.1)$ \\
\hline & $p$ value & 0.007 & 0.763 & 0.123 \\
\hline \multirow[t]{3}{*}{ Gender } & Male & 15.2(12.9-17.9) & $31.2(23.4-40.1)$ & $22.2(15.5-30.6)$ \\
\hline & Female & 13.5(11.7-15.6) & $36.9(29.8-44.6)$ & $17.4(12.4-23.9)$ \\
\hline & $p$ value & 0.287 & 0.320 & 0.314 \\
\hline \multirow[t]{3}{*}{ Residence } & Urban & 19.7(17.5-22.0) & $30.1(24.6-36.2)$ & $15.9(11.7-21.2)$ \\
\hline & Rural & $12.4(10.5-14.5)$ & $36.1(28.3-44.7)$ & $22.3(15.9-30.2)$ \\
\hline & $p$ value & $<0.001$ & 0.237 & 0.130 \\
\hline \multirow[t]{5}{*}{ Education } & Primary school and below & 12.0(9.9-14.5) & $28.9(20.7-38.8)$ & 18.8(11.8-28.6) \\
\hline & Junior middle school & 14.4(12.0-17.2) & $39.6(30.5-49.4)$ & $21.3(14.4-30.4)$ \\
\hline & Senior middle school & $22.3(17.5-27.8)$ & $37.4(25.2-51.6)$ & $17.0(9.6-28.4)$ \\
\hline & College and above & 23.2(17.9-29.6) & $26.1(15.4-40.7)$ & $23.4(13.1-38.3)$ \\
\hline & $p$ value & $<0.001$ & 0.225 & 0.847 \\
\hline \multirow[t]{3}{*}{ Lack of exercise } & Yes & 19.8(16.1-24.1) & $27.0(18.5-37.5)$ & $10.2(5.2-19.0)$ \\
\hline & No & 13.1(11.5-14.8) & $36.4(29.9-43.4)$ & 23.4(17.9-30.0) \\
\hline & $p$ value & 0.001 & 0.128 & 0.013 \\
\hline \multirow[t]{3}{*}{ Smoking } & Yes & 13.6(11.7-15.9) & $31.2(23.9-39.5)$ & $18.8(12.9-26.6)$ \\
\hline & No & 15.5(13.4-18.0) & $37.8(30.2-46.0)$ & $21.4(15.6-28.8)$ \\
\hline & $p$ value & 0.236 & 0.245 & 0.589 \\
\hline \multirow[t]{3}{*}{ Overweight or obesity } & Yes & $20.8(17.9-24.0)$ & $32.1(24.9-40.3)$ & $13.7(8.8-20.7)$ \\
\hline & No & 10.3(8.8-12.1) & $36.2(28.4-44.7)$ & $27.7(20.6-36.1)$ \\
\hline & $p$ value & $<0.001$ & 0.476 & 0.006 \\
\hline \multirow[t]{4}{*}{ Alcohol consumption } & Never & $14.4(12.6-16.3)$ & $37.0(30.6-44.0)$ & $19.7(14.7-25.8)$ \\
\hline & Moderate drinking & $12.1(8.8-16.5)$ & $30.2(16.9-48.0)$ & $27.2(14.8-44.4)$ \\
\hline & Excessive drinking & $17.7(13.5-23.0)$ & $24.1(13.6-39.0)$ & $13.3(5.6-28.6)$ \\
\hline & $p$ value & 0.185 & 0.282 & 0.329 \\
\hline \multirow[t]{3}{*}{ Personal history of stroke } & Yes & $27.4(21.1-34.9)$ & $43.3(29.7-58.0)$ & $18.3(9.2-33.1)$ \\
\hline & No & 13.1(11.6-14.8) & $32.1(26.3-38.5)$ & $20.2(15.4-26.0)$ \\
\hline & $p$ value & $<0.001$ & 0.147 & 0.777 \\
\hline \multirow[t]{3}{*}{ Family history of stroke } & Yes & $15.1(12.6-17.9)$ & $39.6(30.6-49.4)$ & $20.1(13.4-28.8)$ \\
\hline & No & $14.0(12.1-16.0)$ & $30.5(24.1-37.8)$ & $19.8(14.3-26.7)$ \\
\hline & $p$ value & 0.505 & 0.122 & 0.960 \\
\hline \multirow[t]{3}{*}{ Personal history of coronary heart disease } & Yes & $33.2(26.0-41.2)$ & $44.8(31.7-58.7)$ & $29.4(18.2-43.9)$ \\
\hline & No & 12.9(11.4-14.6) & $31.8(25.9-38.3)$ & 18.0(13.4-23.8) \\
\hline & $p$ value & $<0.001$ & 0.080 & 0.078 \\
\hline \multirow[t]{3}{*}{ Family history of coronary heart disease } & Yes & $17.6(14.5-21.2)$ & $43.0(33.0-53.6)$ & $23.7(16.0-33.7)$ \\
\hline & No & 13.2(11.6-15.1) & $29.6(23.5-36.5)$ & $18.1(13.0-24.6)$ \\
\hline & $p$ value & 0.018 & 0.030 & 0.283 \\
\hline \multirow[t]{3}{*}{ Family history of dyslipidemia } & Yes & 15.0(11.8-18.9) & $40.3(28.2-53.9)$ & $17.5(9.7-29.5)$ \\
\hline & No & $14.2(12.5-16.0)$ & $31.7(26.0-37.9)$ & 20.7(15.7-26.8) \\
\hline & $p$ value & 0.683 & 0.224 & 0.595 \\
\hline
\end{tabular}


Table 4 Multivariate logistic regression analyses on influence factors for prevalence of dyslipidemia

\begin{tabular}{|c|c|c|c|c|}
\hline Category & Subcategory & Fully adjusted OR (95\% Cl) & Wald $x^{2}$ value & $p$ value \\
\hline \multirow[t]{4}{*}{ Age(years) } & $40-49$ & 1.00 (Referent) & & \\
\hline & $50-59$ & $1.506(1.277-1.776)$ & 23.739 & $<0.001$ \\
\hline & $60-69$ & $1.524(1.267-1.834)$ & 19.933 & $<0.001$ \\
\hline & $70-$ & $1.335(1.006-1.772)$ & 3.994 & 0.046 \\
\hline \multirow[t]{2}{*}{ Gender } & Female & 1.00 (Referent) & & \\
\hline & Male & $0.914(0.795-1.052)$ & 1.567 & 0.211 \\
\hline \multirow[t]{2}{*}{ Residence } & Rural & 1.00 (Referent) & & \\
\hline & Urban & $1.339(1.161-1.543)$ & 16.184 & $<0.001$ \\
\hline \multirow[t]{2}{*}{ Overweight or obesity } & No & 1.00 (Referent) & & \\
\hline & Yes & $2.156(1.836-2.533)$ & 87.602 & $<0.001$ \\
\hline \multirow[t]{2}{*}{ Lack of exercise } & No & 1.00 (Referent) & & \\
\hline & Yes & $1.212(1.021-1.439)$ & 4.810 & 0.028 \\
\hline \multirow[t]{2}{*}{ Hypertension } & No & 1.00 (Referent) & & \\
\hline & Have & $1.643(1.425-1.893)$ & 47.023 & $<0.001$ \\
\hline \multirow[t]{2}{*}{ Diabetes mellitus } & No & 1.00 (Referent) & & \\
\hline & Have & $2.173(1.661-2.844)$ & 32.006 & $<0.001$ \\
\hline \multirow[t]{2}{*}{ Personal history of coronary heart disease } & No & 1.00 (Referent) & & \\
\hline & Yes & $1.365(1.006-1.851)$ & 3.989 & 0.046 \\
\hline \multirow[t]{2}{*}{ Family history of coronary heart disease } & No & 1.00 (Referent) & & \\
\hline & Yes & $1.182(1.004-1.391)$ & 4.052 & 0.044 \\
\hline \multirow[t]{2}{*}{ Family history of dyslipidemia } & No & 1.00 (Referent) & & \\
\hline & Have & $2.135(1.710-2.666)$ & 44.886 & $<0.001$ \\
\hline
\end{tabular}

Method: Enter: Age, Gender, Residence; Forward-Conditional: Overweight or obesity, Hypertension, Diabetes mellitus, Lack of exercise, Personal history of stroke, Personal history of coronary heart disease, Family history of coronary heart disease, Family history of dyslipidemia

Abbreviations: $O R$ odds ratio, $\mathrm{Cl}$ confidence interval

dyslipidemia (OR $=2.135$; 95\% CI: $1.710,2.666)$ were more likely to have dyslipidemia compared to those without any history of these conditions.

As shown in Table 5, participants living in urban areas $(\mathrm{OR}=1.368$; 95\% CI: $1.021,1.834)$ were more likely to be aware of their dyslipidemia condition than those living in the rural areas. Subjects with education level of senior middle school ( $\mathrm{OR}=1.963$; 95\% CI: $1.305,2.953)$ or college and above $(\mathrm{OR}=2.325$; 95\% CI: $1.447,3.734$ ) tended to be more aware of their dyslipidemia condition than those with education level of primary school and below. Participants overweight or obesity (OR $=1.881 ; 95 \%$ CI: 1.499, 2.359), or those with hypertension $(\mathrm{OR}=1.751$; 95\% CI: $1.339,2.289)$ or diabetes mellitus $(\mathrm{OR}=1.727$; $95 \%$ CI: $1.305,2.286)$ were more inclined to be aware of their dyslipidemia condition. In addition, having a personal history of coronary heart disease $(\mathrm{OR}=2.595$; 95\% CI: $1.864,3.613)$ or stroke $(\mathrm{OR}=2.176$; 95\% CI: $1.552,3.051)$ increased the tendency of dyslipidemia awareness.

Table 6 indicates that subjects with a personal history of coronary heart disease $(\mathrm{OR}=2.021 ; 95 \% \mathrm{CI}: 1.183$, $3.453)$ or stroke $(\mathrm{OR}=1.743$; 95\% CI: $1.002,3.032)$ were more inclined to receive treatment for dyslipidemia than those without these conditions.

Table 7 illustrates that overweight or obesity $(\mathrm{OR}=$ 0.404; $95 \%$ CI: $0.235,0.695)$ and lack of exercise (OR $=0.423 ; 95 \%$ CI: $0.215,0.830)$ were associated with poor control of dyslipidemia; and subjects with personal history of coronary heart disease $(\mathrm{OR}=2.065$; $95 \%$ CI: $1.083,3.905)$ were more likely to control their serum lipids at normal levels.

\section{Discussion}

In this population-based cross-sectional epidemiological study, we have identified a high prevalence of dyslipidemia among adults aged 40 years and over in northeast China, especially in urban areas. Additionally, we found that high triglyceride was the most prevalent type of dyslipidemia in northeast China, followed by high total cholesterol. However, the awareness, treatment, and control rate of dyslipidemia were at far from desirable levels. Increasing age, living in urban regions and family history of dyslipidemia were associated with higher risk of dyslipidemia. But beyond that, participants with underlying chronic diseases, such as overweight or obesity, 
Table 5 Multivariate logistic regression analyses on influence factors for awareness of dyslipidemia

\begin{tabular}{|c|c|c|c|c|}
\hline Category & Subcategory & Fully adjusted OR (95\% Cl) & Wald $x^{2}$ value & $p$ value \\
\hline \multirow[t]{4}{*}{ Age(years) } & $40-49$ & 1.00 (Referent) & & \\
\hline & $50-59$ & $1.133(0.848-1.515)$ & 0.712 & 0.399 \\
\hline & $60-69$ & $1.403(1.023-1.924)$ & 4.419 & 0.036 \\
\hline & $70-$ & $1.221(0.770-1.938)$ & 0.719 & 0.396 \\
\hline \multirow[t]{2}{*}{ Gender } & Female & 1.00 (Referent) & & \\
\hline & Male & $1.011(0.802-1.273)$ & 0.008 & 0.928 \\
\hline \multirow[t]{2}{*}{ Residence } & Rural & 1.00 (Referent) & & \\
\hline & Urban & $1.368(1.021-1.834)$ & 4.398 & 0.036 \\
\hline \multirow[t]{4}{*}{ Education } & Primary school and below & 1.00 (Referent) & & \\
\hline & Junior middle school & $1.252(0.916-1.713)$ & 1.987 & 0.159 \\
\hline & Senior middle school & $1.963(1.305-2.953)$ & 10.487 & 0.001 \\
\hline & College and above & $2.325(1.447-3.734)$ & 12.162 & $<0.001$ \\
\hline \multirow[t]{2}{*}{ Overweight or obesity } & No & 1.00 (Referent) & & \\
\hline & Yes & 1.881(1.499-2.359) & 29.850 & $<0.001$ \\
\hline \multirow[t]{2}{*}{ Hypertension } & No & 1.00 (Referent) & & \\
\hline & Have & 1.751(1.339-2.289) & 16.787 & $<0.001$ \\
\hline \multirow[t]{3}{*}{ Fruit consumption } & $\leq 2 \mathrm{~d} / \mathrm{w}$ & 1.00 (Referent) & & \\
\hline & $3-4 d / w$ & $0.883(0.445-1.753)$ & 0.127 & 0.722 \\
\hline & $\geq 5 \mathrm{~d} / \mathrm{w}$ & $0.319(0.134-0.759)$ & 6.678 & 0.010 \\
\hline \multirow[t]{2}{*}{ Diabetes mellitus } & No & 1.00 (Referent) & & \\
\hline & Have & $1.727(1.305-2.286)$ & 14.621 & $<0.001$ \\
\hline \multirow[t]{2}{*}{ Personal history of coronary heart disease } & No & 1.00 (Referent) & & \\
\hline & Yes & $2.595(1.864-3.613)$ & 31.878 & $<0.001$ \\
\hline \multirow[t]{2}{*}{ Personal history of stroke } & No & 1.00 (Referent) & & \\
\hline & Yes & $2.176(1.552-3.051)$ & 20.314 & $<0.001$ \\
\hline
\end{tabular}

Method: Enter: Age, Gender, Residence; Forward-Conditional: Education, Overweight or obesity, Hypertension, Diabetes mellitus, Fruit consumption, Smoking, Alcohol consumption, Lack of exercise, Personal history of stroke, Personal history of coronary heart disease, Family history of coronary heart disease, Family history of stroke Abbreviations: $O R$ odds ratio, $\mathrm{Cl}$ confidence interval

Table 6 Multivariate logistic regression analyses on influence factors for treatment of dyslipidemia

\begin{tabular}{lllll}
\hline Category & Subcategory & Fully adjusted OR (95\% Cl) & Wald $x^{2}$ value & $p$ value \\
\hline Age(years) & $40-49$ & 1.00 (Referent) & 0.107 & 0.744 \\
& $50-59$ & $1.098(0.627-1.922)$ & 0.143 & 0.088 \\
& $60-69$ & $0.892(0.492-1.615)$ & $1.134(0.494-2.601)$ & 1.435 \\
Gender & $70-$ & 1.00 (Referent) & 0.767 \\
Residence & Female & Male & $0.769(0.500-1.182)$ & 0.750 \\
Personal history of coronary heart disease & Rural & 1.00 (Referent) & 0.231 \\
& Urban & $0.827(0.537-1.272)$ & 6.634 \\
Personal history of stroke & Yes & 1.00 (Referent) & 0.386 \\
& No & $2.021(1.183-3.453)$ & 1.00 (Referent) & 3.873 \\
\hline
\end{tabular}

Method: Enter: Age, Gender, Residence; Forward-Conditional: Personal history of stroke, Personal history of coronary heart disease, Family history of coronary heart disease

Abbreviations: OR odds ratio, $\mathrm{Cl}$ confidence interval 
Table 7 Multivariate logistic regression analyses on influence factors for control of dyslipidemia

\begin{tabular}{|c|c|c|c|c|}
\hline Category & Subcategory & Fully adjusted OR (95\% Cl) & Wald $x^{2}$ value & $p$ value \\
\hline \multirow[t]{4}{*}{ Age(years) } & $40-49$ & 1.00 (Referent) & & \\
\hline & $50-59$ & $0.544(0.264-1.123)$ & 2.707 & 0.100 \\
\hline & $60-69$ & $0.830(0.408-1.687)$ & 0.266 & 0.606 \\
\hline & $70-$ & $1.174(0.458-3.013)$ & 0.112 & 0.738 \\
\hline \multirow[t]{2}{*}{ Gender } & Female & 1.00 (Referent) & & \\
\hline & Male & $1.345(0.787-2.299)$ & 1.178 & 0.278 \\
\hline \multirow[t]{2}{*}{ Residence } & Rural & 1.00 (Referent) & & \\
\hline & Urban & $0.640(0.376-1.091)$ & 2.692 & 0.101 \\
\hline \multirow[t]{2}{*}{ Overweight or obesity } & No & 1.00 (Referent) & & \\
\hline & Yes & $0.404(0.235-0.695)$ & 10.720 & 0.001 \\
\hline \multirow[t]{2}{*}{ Lack of exercise } & No & 1.00 (Referent) & & \\
\hline & Yes & $0.423(0.215-0.830)$ & 6.264 & 0.012 \\
\hline \multirow[t]{2}{*}{ Personal history of coronary heart disease } & No & 1.00 (Referent) & & \\
\hline & Yes & $2.056(1.083-3.905)$ & 4.852 & 0.028 \\
\hline
\end{tabular}

Method: Enter: Age, Gender, Residence; Forward-Conditional: Overweight or obesity, Diabetes mellitus, Fruit consumption, Lack of exercise, Personal history of coronary heart disease, Family history of coronary heart disease

Abbreviations: $O R$ odds ratio, $\mathrm{Cl}$ confidence interval

hypertension, or diabetes mellitus were at a higher risk of dyslipidemia; also these people were more likely to be aware of their condition. However, this did not increase the likelihood of treatment and control of dyslipidemia. Subjects living in urban areas and having higher educational level tended to be more aware of their dyslipidemia condition. Our study also suggests that subjects with personal history of coronary heart disease were most likely to be aware of, treat, and control their dyslipidemia condition; also subjects with personal history of stroke were more likely to be aware of and treat their dyslipidemia condition, but it didn't mean well control of their dyslipidemia. Overweight or obesity and lack of exercise were also associated with poor dyslipidemia control.

The first national study on serum lipids was part of the China National Nutrition and Health Survey performed in 2002 by China's Center for Disease Control and Prevention [17], which showed that the prevalence of dyslipidemia in Chinese adults aged 18 and over was $18.6 \%$, with $17.0,22.9$, and $23.4 \%$ in the age groups of $18-44,45-59$, and over 60 years old, respectively. In this representative sample of northeast Chinese adults aged 40 years and over of our study, the overall prevalence of dyslipidemia was $62.1 \%$, with $58.6 \%, 64.4 \%, 65.7 \%, 63.3 \%$ in the age groups of 40-49, 50-59, 60-69, and over 70 years old, respectively. These prevalence rates in each age group over 40 years were significantly higher than data reported by the China National Survey of Chronic Kidney Disease Working Group, conducted from January 2007 to October $2010[18,19]$. The cross-sectional survey covered 13 provinces but did not contain any provinces in northeast China, reporting that the adjusted prevalence of dyslipidemia in Chinese adults in the age groups of 40-49, $50-59,60-69$, and over 70 years old was 43.6, 46.8, 45.3, and $42.9 \%$, respectively, for males, and $23.5,39.9,50.0$, and $46.6 \%$, respectively, for females. Our study also showed that high triglyceride was the most prevalent form of dyslipidemia in northeast Chinese adults aged 40 years and over, followed by high total cholesterol (the prevalence of high total cholesterol, triglyceride, low-density lipoprotein cholesterol, and low high-density lipoprotein cholesterol were $33.5,43.9,0.6$, and $8.8 \%$, respectively). This was consistent with the overall pooled analysis, including thirty-eight studies on the epidemiology of dyslipidemia in Chinese adults [13]; however, this was different from many other national cross-sectional studies $[18,20,21]$, in which low high-density lipoprotein cholesterol or high low-density lipoprotein cholesterol was the major type of dyslipidemia. In addition to the differences in methodology and population demography, local climate conditions, dietary habits, and sedentary lifestyles might contribute to the high prevalence of dyslipidemia [22], especially high triglyceride and high total cholesterol in the northeast Chinese adults aged 40 years and over. Jilin Province, located in the center of northeast China, has a temperate continental monsoon climate. The cold climate leads to a deficiency of fresh fruits and vegetables; thus, people eat relatively more animal fat and fewer fresh vegetables and fruits. This diet leads to a high prevalence of high triglyceride and total cholesterol. Moreover, the cold weather limits people's outdoor physical activity during the long winter, 
consequently increasing the risk of overweight or obesity and related metabolic abnormalities. Elevated triglycerides [10] and high total cholesterol levels [8], as predictive of cardiovascular risk, have been wellestablished. Therefore, lowering triglyceride and total cholesterol levels is the primary and urgent target for cardiovascular disease prevention in northeast China.

In the present study, factors associated with the prevalence of dyslipidemia included increasing age, living in urban regions, having personal or family history of coronary heart disease, and family history of dyslipidemia. Our study also found that overweight or obesity and lack of exercise were associated with an increased the risk of dyslipidemia. In addition, people with underlying chronic diseases, such as hypertension, or diabetes mellitus were at a higher risk of dyslipidemia. These results were partly consistent with many previous studies [18, 20, 21, 23]. It may reflect that factors associated with dyslipidemia and the consequences of dyslipidemia (such as overweight or obesity) are similar among different ethnicities. This suggests that people with underlying chronic diseases, such as obesity, hypertension, and diabetes mellitus are at a higher risk of developing dyslipidemia and should be more carefully monitored and managed.

Dyslipidemia is one of the most important independent modifiable risk factors for cardiovascular disease in both Western [24, 25] and Asian [26, 27] populations. Raising the awareness, treatment and control rate of dyslipidemia has a positive impact on the primary and secondary prevention of cardiovascular disease. In 2010, $\mathrm{Li}$ et al. [28] reported that the awareness rate of dyslipidemia among Chinese adults aged 45-59 and over 60 years old was 16.75 and $18.74 \%$, respectively. The treatment rate was 10.73 and $12.05 \%$ in the corresponding age groups, and the control rates were was 5.49 and $6.94 \%$, respectively. Our study showed that the awareness, treatment, and control rate of dyslipidemia in northeast Chinese adults aged 40 years and over were $14.4,33.9$, and $19.9 \%$, respectively. These rates were significantly higher than the China National Diabetes and Metabolic Disorders Study [29] conducted from June 2007 to May 2008, in which the awareness, treatment, and control of borderline high or high total cholesterol was $11.0,5.1$, and $2.8 \%$, respectively. This showed that with the great importance attached to early screening, health education, and intervention therapy in recent years, people's awareness and prevention methods for chronic disease are being improved. Even so, these rates are still far below those from Canada and the US [30]. In the current study, we also found that lipid-lowering drugs alone would not solve the problem of dyslipidemia control completely. Even in those who were receiving treatment for dyslipidemia, only $19.9 \%$ participants control their serum lipids at normal levels. In addition to increasing rates of statin prescription and lifestyle intervention in the patients with severe dyslipidemia [31-33], we should popularize the awareness of nutraceuticals and functional food ingredients in influencing dyslipidemia in the public. Scicchitano P et al.[34] suggested that nutraceutical and functional food ingredients, such as resveratrol, polyphenols, proanthocyanidins, fish oil, etc., can be adopted in the common pharmacological treatments for dyslipidemia, such as statin therapy. They can positively influence lipid profile by combining the effects of drug therapy. What's more, they can be considered as an alternative therapeutic option in situations where statins cannot be used because of intolerance.

In this study, we found that subjects with a personal history of coronary heart disease or stroke were more likely to be aware of and treat their dyslipidemia condition. Moreover, subjects with personal history of coronary heart disease were more inclined to control their serum lipids at normal levels, while this was not found in the subjects with personal history of stroke. Previous researches [35-37] have shown that those who had a cardiovascular event before often become more focused on their health, being particularly concerned about cardiovascular disease risk factors, including dyslipidemia, and these patients always were more probably to engage in and comply with lipid-lowering drugs or lifestyle intervention for dyslipidemia. Our study also revealed that those highly educated usually had a higher rate of awareness than those with low education levels. This finding was consistent with several other studies $[14,38-41]$. Previous report has suggested that education is the best socioeconomic status index and predicts awareness of risk factors for cardiovascular disease [42]. The higher the level of education attained by an individual, the more likely they are to express increased awareness about health conditions, including dyslipidemia.

This population-based cross-sectional epidemiologic study was conducted in a large representative sample of northeast Chinese adults aged 40 and over. The data were taken from the 2015-16 Stroke Screening and Prevention Program in northeast China. Standard protocols and instruments designed by the Stroke Screening and Prevention Program of the National Health and Family Planning Commission of China, along with strict training processes for data collection, were used to ensure the high quality of the data. In addition, standard laboratory methods for the measurement of serum lipids were used, and the sample was uniformly measured by Changchun Kingmed Center for Clinical Laboratory Co., Ltd. For all the aforementioned reasons, our study provides the most reliable and up-todate information on the prevalence, awareness, treatment, and control of dyslipidemia in adults aged 40 and over in northeast China. 
However, the findings of our present study should be interpreted with an understanding of the following limitations. First, as these findings were derived from a cross-sectional study without a strict follow-up design, and the prevalence, awareness, treatment, and control of dyslipidemia were based on questionnaire and measurements taken by a single visit, the results are prone to be affected by the recall bias and unmeasured confounding. Second, the definition of dyslipidemia in our study was based on Chinese guidelines on prevention and treatment of dyslipidemia in adults; therefore, the comparison between our results and those from other countries should be carefully made. Finally, no causal relationships could be precisely delineated for the properties of the cross-sectional study.

\section{Conclusions}

In conclusion, we have identified a high prevalence of dyslipidemia among adults aged 40 years and over in northeast China, especially in the urban areas. Our cross-sectional study showed that high triglycerides and high total cholesterol are two major types of dyslipidemia in northeast China. However, the awareness, treatment, and control rates of dyslipidemia are far from the desirable levels. Renewed efforts taking influence factors into account are needed to lower the prevalence of dyslipidemia and increase the awareness, treatment, and control rates of dyslipidemia. These efforts includes strengthening the primary care system, promotion of a healthy lifestyle, and energetically improve people's educational degrees.

\section{Abbreviations}

BMI: Body mass index; Cl: Confidence interval; DBP: Diastolic blood pressure; FBG: Fasting blood-glucose; HDL-C: High-density lipoprotein cholesterol; H-LDL: High low-density lipoprotein cholesterol; H-TC: High total cholesterol; H-TG: High triglycerides; LDL-C: Low-density lipoprotein cholesterol; L-HDL: Low high-density lipoprotein cholesterol; OR: Odds ratio; SBP: Systolic blood pressure; TC: Total cholesterol; TGs: Triglycerides

\section{Acknowledgments}

We gratefully acknowledge all study participants, interviewers from the First Hospital of Jilin University, and the support from the Chinese National Center for Stroke Care Quality Control and Management.

\section{Funding}

This article was supported by the Natural Science Foundation of China (NSFC, Grant No. 8100945).

\section{Availability of data and materials}

The datasets used and/or analyzed during the current study available from the corresponding author on reasonable request.

\section{Authors' contributions}

Conception and design: ZNG, YQX, and YY. Acquisition of data: FLZ, HYL, YL, and MSS. Analysis: FLZ and YHW. Drafting the manuscript: FLZ. Critical revision: ZNG, YY. All authors approved the final version to be published.

\section{Competing interests}

The authors declare that they have no competing interests.
Consent for publication

Not applicable.

\section{Ethics approval and consent to participate}

The study design was approved by the Human Ethics and Research Ethics committees of the First Hospital of Jilin University (Approval Number: 2015-R-250). Written informed consent was obtained from the participants in the survey.

\section{Publisher's Note}

Springer Nature remains neutral with regard to jurisdictional claims in published maps and institutional affiliations.

\section{Author details}

'Stroke Center, Department of Neurology, the First Hospital of Jilin University, Xinmin Street 71\#, 130021 Changchun, China. ${ }^{2}$ Neuroscience Center, Department of Neurology, the First Hospital of Jilin University, Xinmin Street 71\#, 130021 Chang Chun, China. ${ }^{3}$ Division of Clinical Research, the First Hospital of Jilin University, Changchun, China.

Received: 15 December 2016 Accepted: 12 March 2017

Published online: 23 March 2017

\section{References}

1. Murray CJ, Barber RM, Foreman KJ, Abbasoglu Ozgoren A, Abd-Allah F, Abera SF, Aboyans V, Abraham JP, Abubakar I, Abu-Raddad L, et al. Global, regional, and national disability-adjusted life years (DALYs) for 306 diseases and injuries and healthy life expectancy (HALE) for 188 countries, 1990-2013: quantifying the epidemiological transition. Lancet. 2015;386:2145-91.

2. BD 2013 Mortality and Causes of Death Collaborators. Global, regional, and national age-sex specific all-cause and cause-specific mortality for 240 causes of death, 1990-2013: a systematic analysis for the Global Burden of Disease Study 2013. Lancet. 2015;385:117-71.

3. Stevens W, Peneva D, Li JZ, Liu LZ, Liu G, Gao R, Lakdawalla DN. Estimating the future burden of cardiovascular disease and the value of lipid and blood pressure control therapies in China. BMC Health Serv Res. 2016;16:175.

4. Kim AS, Cahill E, Cheng NT. Global stroke belt: geographic variation in stroke burden worldwide. Stroke. 2015;46:3564-70.

5. Wu Y, Benjamin EJ, MacMahon S. Prevention and control of cardiovascular disease in the rapidly changing economy of china. Circulation. 2016; 133:2545-60.

6. Feigin VL, Roth GA, Naghavi M, Parmar P, Krishnamurthi R, Chugh S, Mensah GA, Norrving B, Shiue I, Ng M, et al. Global burden of stroke and risk factors in 188 countries, during 1990-2013: a systematic analysis for the global burden of disease study 2013. Lancet Neurol. 2016;15:913-24.

7. Levenson JW, Skerrett PJ, Gaziano JM. Reducing the global burden of cardiovascular disease: the role of risk factors. Prev Cardiol. 2002;5:188-99.

8. Expert Panel on Detection, Evaluation, and Treatment of High Blood Cholesterol in Adults. Executive summary of the third report of the national cholesterol education program (NCEP) expert panel on detection, evaluation, and treatment of high blood cholesterol in adults (adult treatment panel III). JAMA. 2001;285:2486-97.

9. Castelli WP. Cholesterol and lipids in the risk of coronary artery disease-the Framingham heart study. Can J Cardiol. 1988:4(Suppl A):5a-10a.

10. Sarwar N, Danesh J, Eiriksdottir G, Sigurdsson G, Wareham N, Bingham S, Boekholdt SM, Khaw KT, Gudnason V. Triglycerides and the risk of coronary heart disease: 10,158 incident cases among 262,525 participants in 29 Western prospective studies. Circulation. 2007;115:450-8.

11. Strategy TCSGoToCDiCaP. Current status of major cardiovascular risk factors in Chinese populations and their trends in the past two decades. Chin J Cardiol. 2001:29:74-9.

12. Farzadfar F, Finucane MM, Danaei G, Pelizzari PM, Cowan MJ, Paciorek CJ, Singh GM, Lin JK, Stevens GA, Riley LM, Ezzati M. National, regional, and global trends in serum total cholesterol since 1980: systematic analysis of health examination surveys and epidemiological studies with 321 country-years and 3.0 million participants. Lancet. 2011;377:578-86.

13. Huang Y, Gao L, Xie X, Tan SC. Epidemiology of dyslipidemia in Chinese adults: meta-analysis of prevalence, awareness, treatment, and control. Popul Health Metr. 2014;12:28.

14. He H, Yu YQ, Li Y, Kou CG, Li B, Tao YC, Zhen Q, Wang C, Kanu JS, Huang $X F$, et al. Dyslipidemia awareness, treatment, control and influence factors 
among adults in the Jilin province in China: a cross-sectional study. Lipids Health Dis. 2014;13:122.

15. Sun H, Zou X, Liu L. Epidemiological factors of stroke: a survey of the current status in china. J Stroke. 2013:15:109-14.

16. Joint Committee for Developing Chinese guidelines on Prevention and Treatment of Dyslipidemia in Adults. Chinese guidelines on prevention and treatment of dyslipidemia in adults. Zhonghua Xin Xue Guan Bing Za Zhi. 2007;35:390-419.

17. Zhao WHZJ, You Y, Man QQ, Li H, Wang CR, Zhai Y, Li Y, Jin SG, Yang XG. Epidemiologic characteristics of dyslipidemia in people aged 18 years and over in China. Zhonghua Yu Fang Yi Xue Za Zhi. 2005;39:306-10.

18. Pan L, Yang Z, Wu Y, Yin RX, Liao Y, Wang J, Gao B, Zhang L. The prevalence, awareness, treatment and control of dyslipidemia among adults in China. Atherosclerosis. 2016;248:2-9.

19. Zhang L, Wang F, Wang L, Wang W, Liu B, Liu J, Chen M, He Q, Liao Y, Yu X, et al. Prevalence of chronic kidney disease in China: a cross-sectional survey. Lancet. 2012;379:815-22

20. Erem C, Hacihasanoglu A, Deger O, Kocak M, Topbas M. Prevalence of dyslipidemia and associated risk factors among Turkish adults: Trabzon lipid study. Endocrine. 2008;34:36-51.

21. Joshi SR, Anjana RM, Deepa M, Pradeepa R, Bhansali A, Dhandania VK, Joshi PP, Unnikrishnan R, Nirmal E, Subashini R, et al. Prevalence of dyslipidemia in urban and rural India: the ICMR-INDIAB study. PLoS One. 2014;9:e96808.

22. Zhou B, Rao X, Dennis BH, Li Y, Zhuo Q, Folsom AR, Yang J, Li Y, Stamler J, Cao $T$, et al. The relationship between dietary factors and serum lipids in Chinese urban and rural populations of Beijing and Guangzhou. PRC-USA Cardiovascular and Cardiopulmonary Research Group. Int J Epidemiol. 1995;24:528-34.

23. Wang $S, X u L$, Jonas JB, You QS, Wang YX, Yang H. Prevalence and associated factors of dyslipidemia in the adult Chinese population. PLoS One. 2011;6:e17326.

24. Stamler J, Daviglus ML, Garside DB, Dyer AR, Greenland P, Neaton JD. Relationship of baseline serum cholesterol levels in 3 large cohorts of younger men to long-term coronary, cardiovascular, and all-cause mortality and to longevity. JAMA. 2000;284:311-8.

25. LaRosa JC, He J, Vupputuri S. Effect of statins on risk of coronary disease: a meta-analysis of randomized controlled trials. JAMA. 1999;282:2340-6.

26. Chen Z, Peto R, Collins R, MacMahon S, Lu J, Li W. Serum cholesterol concentration and coronary heart disease in population with low cholesterol concentrations. BMJ. 1991;303:276-82.

27. Blood pressure, cholesterol, and stroke in eastern Asia. Eastern Stroke and Coronary Heart Disease Collaborative Research Group. Lancet. 1998; 352: 1801-1807.

28. Li JH, Wang LM, Mi SQ, Zhang M, Li YC, Jiang Y, Xu Y, Dai M, Wang LH. Awareness rate, treatment rate and control rate of dyslipidemia in Chinese adults, 2010. Zhonghua Yu Fang Yi Xue Za Zhi. 2012;46:687-91.

29. Yang W, Xiao J, Yang Z, Ji L, Jia W, Weng J, Lu J, Shan Z, Liu J, Tian H, et al. Serum lipids and lipoproteins in Chinese men and women. Circulation. 2012;125:2212-21.

30. Alabousi M, Abdullah P, Alter DA, Booth GL, Hogg W, Ko DT, Manuel DG, Farkouh ME, Tu JV, Udell JA. Cardiovascular Risk Factor Management Performance in Canada and the United States: A Systematic Review. Can J Cardiol. 2017;33:393-404.

31. Al-Kindi SG, DeCicco A, Longenecker CT, Dalton J, Simon DI, Zidar DA. Rate of Statin Prescription in Younger Patients With Severe Dyslipidemia. JAMA Cardiol. 2017. doi:10.1001/jamacardio.2016.5162.

32. Arao T, Oida Y, Maruyama C, Mutou T, Sawada S, Matsuzuki H, Nakanishi Y. Impact of lifestyle intervention on physical activity and diet of Japanese workers. Prev Med. 2007:45:146-52.

33. Al-Mahmood AK, Ismail AA, Rashid FA, Azwany YN, Singh R, Gill G. Effect of therapeutic lifestyle changes on insulin sensitivity of non-obese hyperlipidemic subjects: preliminary report. J Atheroscler Thromb. 2007;14:122-7.

34. Scicchitano P, Cameli M, Maiello M, Modesti PA, Muiesan ML, Novo S, Palmiero P, Saba PS, Pedrinelli R, Ciccone MM. Nutraceuticals and dyslipidaemia: beyond the common therapeutics. J Funct Foods. 2014;6:11-32.

35. Nolte $\mathrm{CH}$, Jungehulsing GJ, Rossnagel K, Roll S, Haeusler KG, Reich A, Willich SN, Villringer A, Muller-Nordhorn J. Vascular risk factor awareness before and pharmacological treatment before and after stroke and TIA. Eur J Neurol. 2009;16:678-83.

36. Muntner P, DeSalvo KB, Wildman RP, Raggi P, He J, Whelton PK. Trends in the prevalence, awareness, treatment, and control of cardiovascular disease risk factors among noninstitutionalized patients with a history of myocardial infarction and stroke. Am J Epidemiol. 2006;163:913-20.

37. Kirkland SA, MacLean DR, Langille DB, Joffres MR, MacPherson KM, Andreou $P$. Knowledge and awareness of risk factors for cardiovascular disease among Canadians 55 to 74 years of age: results from the Canadian Heart Health Surveys, 1986-1992. CMAJ. 1999;161:S10-16.

38. McDonald M, Hertz RP, Unger AN, Lustik MB. Prevalence, awareness, and management of hypertension, dyslipidemia, and diabetes among United States adults aged 65 and older. J Gerontol A Biol Sci Med Sci. 2009;64:256-63.

39. Lotufo PA, Santos RD, Figueiredo RM, Pereira AC, Mill JG, Alvim SM, Fonseca MJ, Almeida MC, Molina MC, Chor D, et al. Prevalence, awareness, treatment, and control of high low-density lipoprotein cholesterol in Brazil: Baseline of the Brazilian Longitudinal Study of Adult Health (ELSA-Brasil). J Clin Lipidol. 2016;10:568-76.

40. Redondo A, Benach J, Subirana I, Martinez JM, Munoz MA, Masia R, Ramos R, Sala J, Marrugat J, Elosua R. Trends in the prevalence, awareness, treatment, and control of cardiovascular risk factors across educational level in the 1995-2005 period. Ann Epidemiol. 2011;21:555-63.

41. Frontini MG, Srinivasan SR, Elkasabany A, Berenson GS. Awareness of hypertension and dyslipidemia in a semirural population of young adults: the Bogalusa Heart Study. Prev Med. 2003;36:398-402.

42. Winkleby MA, Jatulis DE, Frank E, Fortmann SP. Socioeconomic status and health: how education, income, and occupation contribute to risk factors for cardiovascular disease. Am J Public Health. 1992:82:816-20.

\section{Submit your next manuscript to BioMed Central and we will help you at every step:}

- We accept pre-submission inquiries

- Our selector tool helps you to find the most relevant journal

- We provide round the clock customer support

- Convenient online submission

- Thorough peer review

- Inclusion in PubMed and all major indexing services

- Maximum visibility for your research

Submit your manuscript at www.biomedcentral.com/submit
Biomed Central 